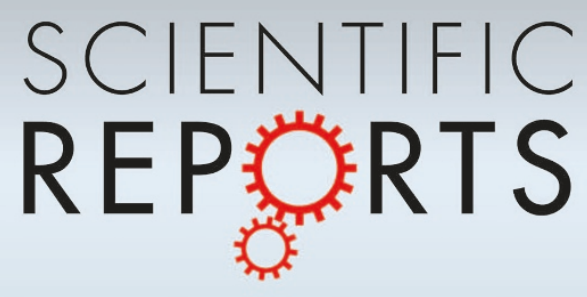

OPEN

SUBJECT AREAS:

COMPUTATIONAL

BIOPHYSICS

NANOSCALE BIOPHYSICS

CARBON NANOTUBES AND

FULLERENES

Received

6 February 2014

Accepted

13 June 2014

Published

17 July 2014

Correspondence and requests for materials should be addressed to T.A.H. (tamsyn.hilder@ anu.edu.au)

\section{Binding of fullerenes and nanotubes to MscL}

\author{
Tamsyn A. Hilder' , Pietro Ridone², Yoshitaka Nakayama², Boris Martinac ${ }^{2,3} \&$ Shin-Ho Chung'
}

${ }^{1}$ Computational Biophysics Group, Research School of Biology, Australian National University, ACT 0200, Australia, ${ }^{2}$ Molecular Cardiology and Biophysics Division, Victor Chang Cardiac Research Institute, NSW 2010, Australia, ${ }^{3}$ St Vincent's Clinical School, The University of New South Wales, Victoria St, St Vincent's Hospital Darlinghurst NSW 2010, Australia.

Multi-drug resistance is becoming an increasing problem in the treatment of bacterial infections and diseases. The mechanosensitive channel of large conductance $(\mathrm{MscL})$ is highly conserved among prokaryotes. Evidence suggests that a pharmacological agent that can affect the gating of, or block the current through, MscL has significant potential as a new class of antimicrobial compound capable of targeting a range of pathogenic bacteria with minimal side-effects to infected patients. Using molecular dynamics we examine the binding of fullerenes and nanotubes to MscL and demonstrate that both are stable within the MscL pore. We predict that fullerenes will attenuate the flow of ions through MscL by reducing the pore volume available to water and ions, but nanotubes will prevent pore closure resulting in a permanently open pore. Moreover, we confirm experimentally that it is possible to attenuate the flow of ions through MscL using a $\mathrm{C}_{60}-\gamma$ cyclodextrin complex.

he Mechanosensitive channel of Small conductance (MscS) and Large conductance (MscL) act as biological emergency release valves which sense tension within the membrane ${ }^{1-6}$. These channels are indispensable for bacterial cell survival when the external environment becomes hypo-osmotic relative to the cell interior causing an increase in cellular turgor sufficient to lyse the microbe ${ }^{3}$. They open in response to the membrane tension so as to relieve pressure and thus prevent membrane damage, and are inactivated once osmotic equilibrium is restored ${ }^{5}$. The presence of either of these channels is sufficient to confer significant protection against hypo-osmotic stress ${ }^{7}$, but mutants lacking both channels are osmotically fragile during hypo-osmotic shock ${ }^{1,3,7}$ and tend to lyse when confronted with a sudden hypotonic shock ${ }^{2}$. In other words, the absence of one of the channels can be compensated with the other ${ }^{8}$. For further details we refer the reader to a number of reviews on mechanosensitive channels ${ }^{1-3,6,9}$.

MscL is wide-spread among bacteria with currently 2296 members of the MscL subfamily listed in the UniProt database ${ }^{10}$; some MscL are found in archaea and possibly in fungi ${ }^{2,10}$. It is the largest gated channel known with a conductance of approximately $3.6 \mathrm{nS}^{1}$ owing to its very large pore diameter $(\geq 30 \AA)^{3,5,7}$. In comparison, MscS has a diameter of approximately $14-16 \AA$ and conductance of $1 \mathrm{nS}^{7}$. The threshold pressure required for activation by membrane tension is 1.5 times higher for MscL than $\mathrm{MscS}$ and it is non-selective for both anions and cations. The narrowest point along the pore forms the channel gate and is approximately $2 \AA$ in diameter in the closed $\mathrm{channel}^{8}$. It is formed by hydrophobic residues, five Val and five Ile that are highly conserved among bacterial members of the MscL family ${ }^{8}$. In particular, the narrowest constriction is formed by the Val-21 residue in the crystal structure of Steinbacher et al. ${ }^{11}$.

Multi-drug resistance in pathogenic strains of bacteria has, in the last decade, presented an increasing problem in the treatment of bacterial infections and diseases ${ }^{8}$. A pharmacological agent that can affect the gating of MscL could lead to impairment of bacterial growth or cell death and a possible new class of antimicrobial agents ${ }^{12,13}$, potentially targeting a range of pathogenic bacteria with minimal side-effects to infected patients ${ }^{8,10}$. The potential of developing antimicrobial agents targeting MscL channels has already been demonstrated by a number of researchers. GsMTx4, a 34-residue peptide isolated from tarantula venom, was the first specific reagent of mechanosensitive channels ${ }^{9}$, and acts by sensitizing both MscS and MscL to membrane tension by penetrating the bilayer ${ }^{8,9,14,15}$. GsMTx4 is an amphipathic compound, with both hydrophobic and hydrophilic regions. These types of compounds affect mechanosensitive channels by altering the local membrane curvature/tension ${ }^{8,9}$ and have been shown to thin the membrane in recent molecular dynamics (MD) studies ${ }^{15}$. Non-specific inhibitors of mechanosensitive channels have also been identified, for example, gadolinium (10-20 $\mu \mathrm{M})$, amiloride and some of its derivatives $(20-100 \mu \mathrm{M})$, and cationic antibiotics such as streptomycin ${ }^{9}$. Moreover, parabens have been identified as possible antimicrobial agents ${ }^{8,13}$. Nguyen et al. $^{13}$ found that propyl and ethyl paraben spontaneously 
activate MscL, and that propyl paraben caused an increase in MscL activity, a lowering of the pressure required to open the pore $50 \%$ of the time and a lowering of the energy required to open the channel. Using Autodock they showed that propyl paraben binds to the channel gate of $\mathrm{MscL}$ at residue Ala-20 with an energy of $-4.91 \mathrm{kcal} /$ $\mathrm{mol}^{13}$. Their results indicate that the interaction between parabens and MscL and MscS inhibit growth of bacteria by opening the channels and thus collapsing the cell turgor and allowing leakage of cytoplasmic contents ${ }^{13}$. In addition, eriochrome cyanine $\mathrm{R}$, a triphenylmethane (TFM) dye used mostly for determination of aluminium by diffusion reflection spectroscopy, was recently reported as a compound that could bind to the hydrophobic gate of MscL with energy of $-11.27 \mathrm{kcal} / \mathrm{mol}$, which is sufficient to induce frequent and relatively long spontaneous openings of $\mathrm{MscL}^{10,16}$.

In this paper we examine the binding of two fullerenes and three nanotubes to $\mathrm{MscL}$ using equilibrium molecular dynamics (MD) simulations. We examine whether such molecules could be further investigated as new antimicrobial agents. We demonstrate and confirm experimentally that fullerenes may be capable of attenuating ionic currents through MscL and result in a slower recovery from osmotic downshock. In contrast, we demonstrate theoretically that nanotubes may prevent pore closure and cause the cell to continually leak cytoplasmic contents.

\section{Results}

Simulation results. Equilibration of MscL open pore model. After 32 ns of unrestrained MD simulations, the open pore model of $\mathrm{MscL}^{17}$ is seen to partially close when compared to the closed crystal structure of $\mathrm{Tb}-\mathrm{MscL}$, as shown in Figure 1A. The pore is closed at the intracellular end and aligns with the closed crystal structure, but remains partially open at the centre of the pore (pore constriction/gate). Our partially closed model has a radius of $5.2 \AA$ at the constricted pore region lined by hydrophobic residues compared to $0.9 \AA$ and $13.4 \AA$ in the closed and open models. The measurement of the radius is taken at the position of the Val-21 residues in each model. The approximate position of Val-21 on the open model is indicated in Figure $1 \mathrm{~B}^{17}$. Thus, the radius of the protein at the pore gate decreases by approximately $8.2 \AA$. The most significant portion of pore closure ( $5 \AA$ decrease) occurs in the first ns, where the pore radius decreases from 13.4 to $8.4 \AA$. In FRET spectroscopy the radius of the pore of Ec-MscL is found to increase by $8 \AA$ upon channel activation ${ }^{18}$. Narrow hydrophobic pores can prevent the passage of ions without presenting a physical occlusion, as water tends to evacuate the hydrophobic regions of narrow pores ${ }^{3}$. For model pores this critical radius is approximately $4-4.5 \AA^{3}$. After $32 \mathrm{~ns}$, water is still present in the hydrophobic region of the pore in our partially closed model. Figure 1A also highlights the decrease in inplane area of the protein and channel lengthening which occur upon pore closure $e^{2,3}$.

Spontaneous closure of the MscS pore has been observed by Sotomayor and Schulten using unrestrained MD simulations ${ }^{19}$. We were unable to run our simulations long enough to observe complete pore closure, but did observe a continual decrease in radius with increasing time. However, the radial decrease is slow; from 28 to 32 ns the pore radius decreased by only $0.75 \AA$. Similarly, running for a further $8 \mathrm{~ns}$ (total $40 \mathrm{~ns}$ simulation time) decreased by $1.5 \AA$. It is expected that with longer simulation times the pore would eventually decrease below this critical radius and water would evacuate from this region.

Ions and solutes access the conduction pathway in the transmembrane domain in the open MscL channel through multiple openings (or portals) present in the cytoplasmic domain ${ }^{3}$, located just before the pore narrows (approximately $-20 \AA$ ) at the C-terminal domain. Similar portals are also observed in $\mathrm{MscS}^{2,7}$. The C-terminal domain is stably associated in both the closed and open channel conformations ${ }^{3}$, as shown in Figure 1A.

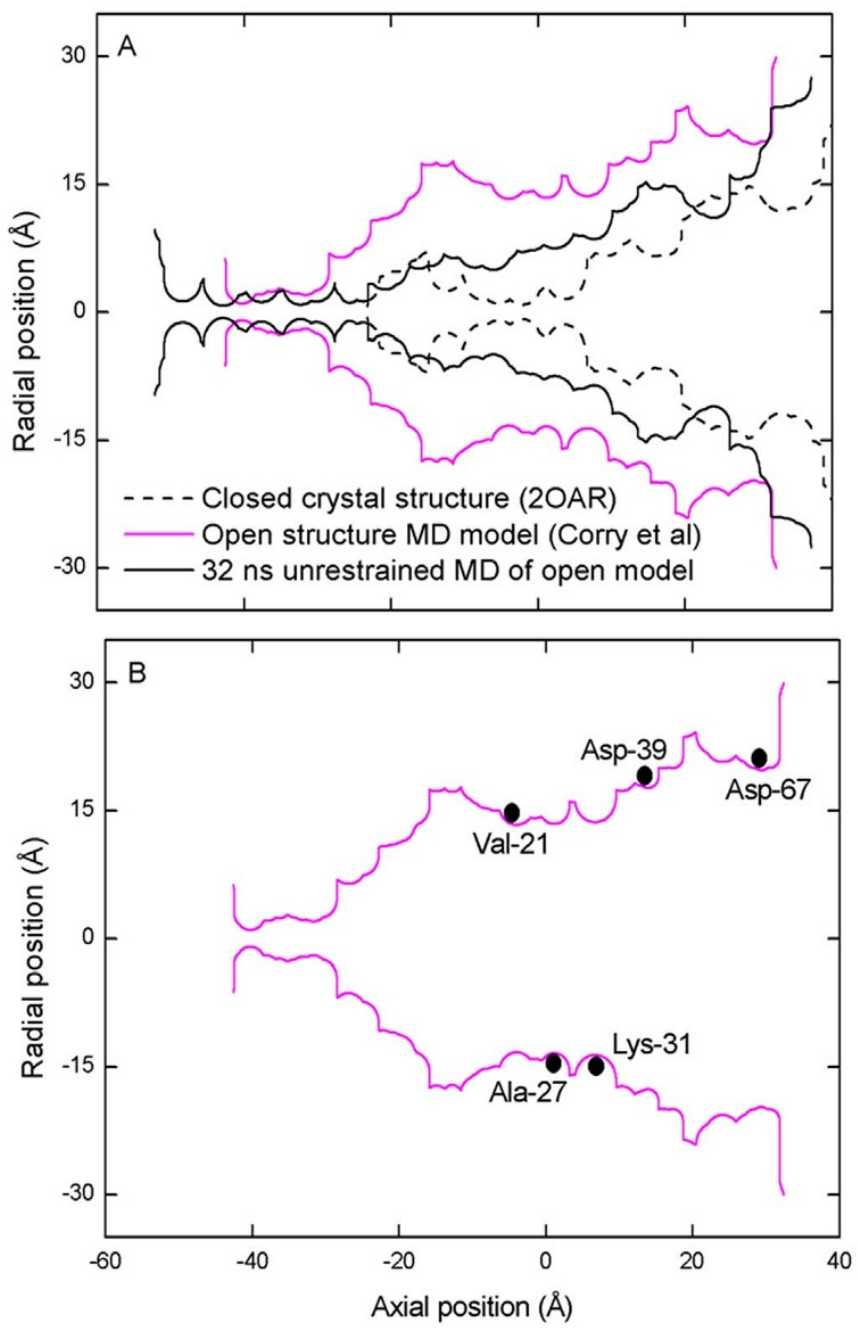

Figure $1 \mid$ (A) MscL pore profile. Shown are the closed crystal structure ${ }^{11,20}$, open all-atom model (Corry et al. ${ }^{17}$ ), and our partially closed model from the 32 ns of unrestrained MD simulations. (B) open all-atom model ${ }^{17}$ with a selection of important residues indicated. The residue labels in (B) have been placed at their average axial location along the length of the pore, and therefore indicate the position of these residues in all subunits.

Fullerenes and the closed pore. We examined two fullerene structures in $\mathrm{MD}$ simulations of the closed pore, a $\mathrm{C}_{60}$ fullerene and a $\mathrm{C}_{60}(\mathrm{OH})_{24}$ fullerene. Both fullerenes are found to move off centre and closer to the pore wall. The fullerenes bind between two of the subunits just outside the tightly constricted region of the pore at $(r, z)$ positions of approximately $(2.0,8.8)$ and $(3.9,9.3) \AA$ for the $\mathrm{C}_{60}$ and $\mathrm{C}_{60}(\mathrm{OH})_{24}$ fullerenes, respectively. These positions are in closest proximity to the Ala-27 and Lys-31 pore residues (indicated in Figure $1 \mathrm{~B}$ ) for the $\mathrm{C}_{60}$ and $\mathrm{C}_{60}(\mathrm{OH})_{24}$ fullerenes, respectively. Figure 2 illustrates the position of the fullerenes relative to Ala-27 and Lys-31. The $\mathrm{C}_{60}$ fullerene is situated closer to the $\mathrm{CHA}$ and $\mathrm{CHB}$ subunits (Figure 2A), whereas the $\mathrm{C}_{60}(\mathrm{OH})_{24}$ fullerene is situated closer to the $\mathrm{CHD}$ and $\mathrm{CHE}$ subunits (Figure $2 \mathrm{~B}$ ).

Fullerenes and the open pore. In all simulations of the fullerenes and the open pore, regardless of initial position, the single $\mathrm{C}_{60}$ fullerene moves off centre and towards the pore wall. The fullerene then binds between two subunits ( $\mathrm{CHB}$ and $\mathrm{CHC}$ ) at a location which is in the same plane as the Val-21 residues (indicated in Figure 1B) and, once bound stays bound. As a result of the symmetry of the pore, it is possible for the fullerene to bind between any two subunits. The 


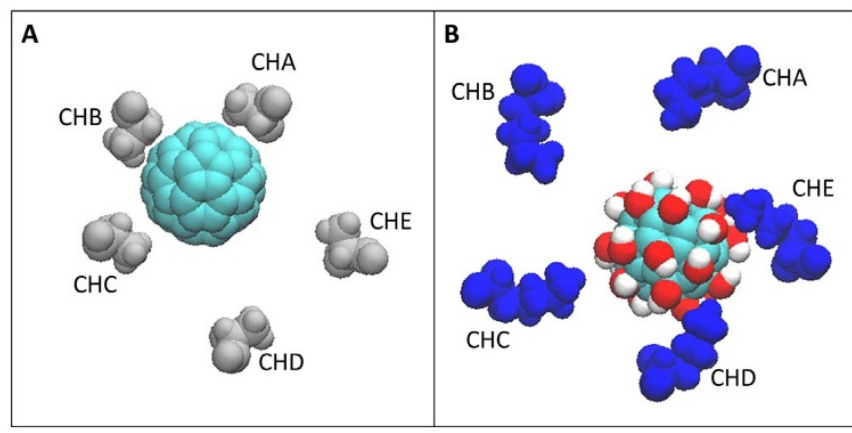

Figure $2 \mid$ Fullerene position in the closed pore. Top view (viewed from extracellular towards intracellular) of the $(\mathrm{A}) \mathrm{C}_{60}$ and $(\mathrm{B}) \mathrm{C}_{60}(\mathrm{OH})_{24}$ fullerenes bound to the Ala-27 and Lys-31 residues. Ala-27 residues are shown in grey and Lys-31 residues are shown in dark blue for all subunits. Note that the $x-y-z$ orientation is identical in both (A) and (B).

fullerene is in a binding pocket surrounded by hydrophobic residues: Phe-29, Ala-20, Leu-19, and Gly-26. After 6 ns the pore radius decreases to $6.26 \AA$ at the location of the Val-21 residues, but remains partially open when compared to the closed crystal structure, as shown in Figure 3A. The partially closed pore profile when the fullerene is bound is slightly different to the profile when the fullerene is absent (Figure 1). When the fullerene is bound the pore dimples slightly on the extracellular side of the fullerene. As mentioned in the methods section, the diameter of the fullerene is approximately $7 \AA$. Therefore, if only one $\mathrm{C}_{60}$ fullerene was present in the pore, the effective pore diameter at this location would be $5.52 \AA$. The fullerene, initially placed on the pore axis at $z=10 \AA$, is located in the same plane as the Val-21 residues and interacts with the residues Ala27, Val-23, and Leu-19. However, the fullerenes initially placed on the pore axis at $\mathrm{z}=5$ and $20 \AA$, are located in the same plane as the Ser-34 residues (slightly above the hydrophobic constriction). In these positions the fullerenes are also interacting with hydrophobic residues, namely Val-23, Ile-24 and Ala-27.

It is reasonable to assume that the presence of multiple $\mathrm{C}_{60}$ fullerenes would be sufficient to block the pore. In simulations with two $\mathrm{C}_{60}$ fullerenes present, the fullerenes bind at a location in the same plane as the Val-21 residues, with one between subunits $\mathrm{CHA}$ and $\mathrm{CHB}$, and the other between subunits $\mathrm{CHB}$ and $\mathrm{CHC}$. We expect that each additional fullerene will bind between a vacant subunit until all five positions are occupied. It is possible that subsequent addition of fullerenes would result in agglomeration inside the pore, and eventually pore blockage.

Similarly, the $\mathrm{C}_{60}(\mathrm{OH})_{24}$ fullerene moves off centre towards the pore wall. Again the fullerene binds between two subunits and, once bound, remains in this position. However, the bound position is located higher up the pore and is in approximately the same plane as the Asp-39 residues (indicated in Figure 1B). The polyhydroxy fullerene is in a binding pocket surrounded by hydrophilic residues: Lys-31, Ser-34, Ser-35 and Asp-39. After $6 \mathrm{ns,} \mathrm{the} \mathrm{pore} \mathrm{radius}$ decreases to $4.44 \AA$ at the location of the Val-21 residues, but remains partially open when compared to the closed crystal structure, as shown in Figure 3B. Again, although the pore diameter decreases, the pore remains open throughout the simulation, as shown in Figure 3B. However, at radii below approximately 4-4.5 $\AA$ hydrophobic pores can prevent the passage of ions without presenting a physical occlusion ${ }^{3}$. Therefore, it is possible that the $\mathrm{C}_{60}(\mathrm{OH})_{24}$ fullerene would significantly reduce or eliminate ionic conduction through MscL. The polyhydroxyl fullerene with an initial position of $z=30 \AA$, does not reach the site in-plane with Asp-39, and is instead located closer to the extracellular reservoir, roughly in the same plane as the Pro-69 residues. The polyhydroxyl fullerene moves further into the pore, closer to the plane formed by the Asp-39
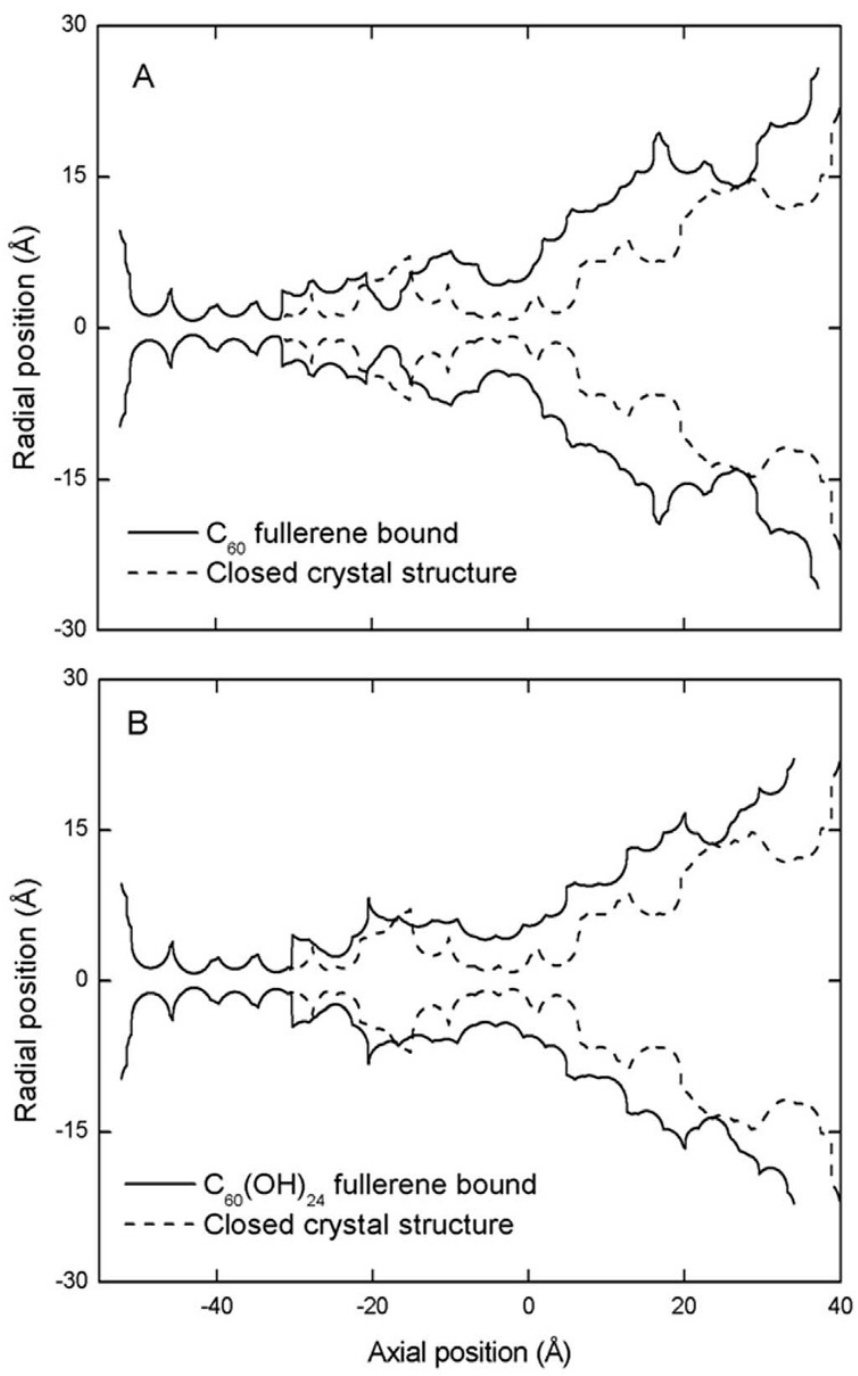

Figure $3 \mid$ MscL pore profile when (A) $\mathrm{C}_{60}$ fullerene and (B) $\mathrm{C}_{60}(\mathrm{OH})_{24}$ fullerene is bound to the pore compared to the closed crystal structure $^{11,20,37}$.

residues with increasing simulation time. Similarly, in simulations with two $\mathrm{C}_{60}(\mathrm{OH})_{24}$ fullerenes present the fullerenes do not reach the site in-plane with the Asp-39 residues, but are located at the extracellular entrance to the pore, and interact with each other and the Asp-67 residues (indicated in Figure 1B) of subunit CHA and CHE.

Nanotubes and an open pore. We examined three nanotube structures in MD simulations of the open pore, a $(18,18)$ boron nitride nanotube $(\mathrm{BNT})$, and a $(18,18)$ and $(14,14)$ carbon nanotube $(\mathrm{CNT})$ all with a length of approximately $20 \AA$. All three nanotubes move further into the pore in MD simulations. After 4 ns the centre of mass of the BNT has moved from $(0,0,9)$ to $(0,0,-7.6)$. All subunits participate in the interaction with the BNT, and residues 13 to 34 wrap around the surface of the BNT. Of particular interest are Arg-13 and Ser-34 which are located at either end of the BNT, as illustrated in Figure 4A. The atom-atom distance between both the nitrogen atom (NH2) of Arg-13 residues and positively charged boron atoms of the BNT, and the oxygen atom of Ser-34 (OG) residues and the nitrogen atoms of the BNT is $<4 \AA$. The pore outline straightens in the vicinity of the BNT as a result of nearby residues wrapping tightly around the nanotube surface, as illustrated in Figure 4B. We predict that the BNT will prevent MscL from closing, and result in a permanently open pore. Alternatively, a capped BNT would result in a permanently blocked pore. 

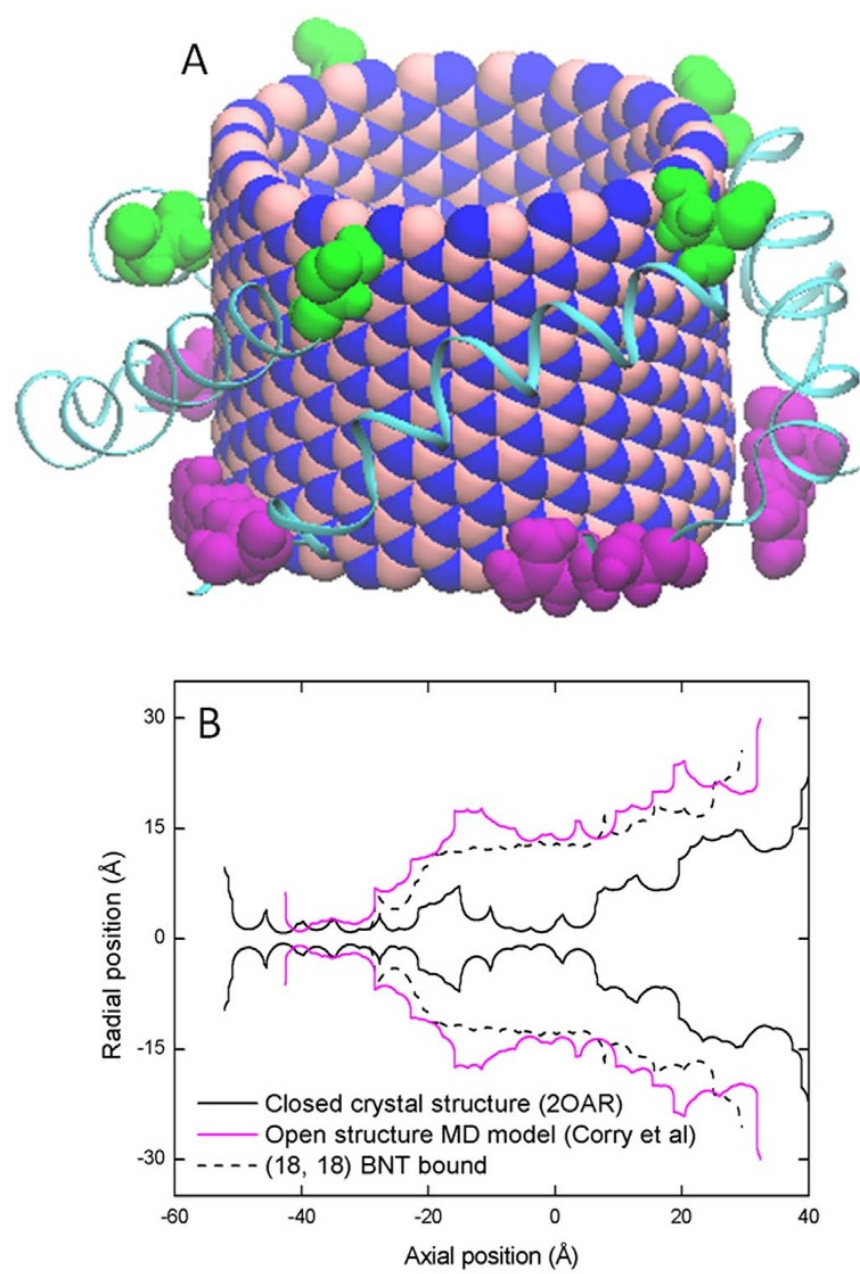

Figure $4 \mid(18,18)$ boron nitride nanotube (BNT) and MscL. (A) Snapshot from MD simulations of the BNT within the MscL pore. Boron and nitrogen atoms of the BNT are shown in light pink and dark blue, respectively. Residues from 13 to 34 wrap around the nanotube surface, and are illustrated as ribbons. Residue Arg-13 and Ser-34 are highlighted in purple and green, respectively. (B) MscL pore profile when the BNT is bound compared to the closed crystal structure $\mathrm{e}^{11,20,37}$ and open all-atom $\operatorname{model}^{17}$.
Similarly, after 4 ns the centre of mass of the $(18,18)$ CNT has moved from $(0,0,9)$ to $(0,0,-7.6)$, and all subunits (residues 13 to 34) wrap around the surface of the CNT. In contrast to the BNT, hydrophobic residues in this region are interacting with the hydrophobic CNT surface: Leu-19, Val-23, Val-33, Ala-27, and Gly-30. Figure 5A and 5B illustrate the hydrophobic residues interacting with the nanotube surface. Again, the pore collapses to tightly wrap around the nanotube surface, and a similar pore outline as the BNT is observed.

For the narrower diameter of the $(14,14)$ CNT (by approximately $5 \AA$ ), the MscL pore again collapses to tightly wrap around the nanotube surface and the pore outline straightens in its vicinity (as shown in Figure 5C). Similar to the other nanotubes examined, the nanotube moves further into the pore, but also moves slightly off centre (towards the $\mathrm{CHA}$ and $\mathrm{CHB}$ subunits) from $(0,0,9)$ to approximately $(0.2,-1.6,-6.8)$. Again, residues 13 to 34 of the protein wrap tightly around the nanotube surface, and the interacting hydrophobic residues are Val-23, Ala-27, Leu-19 and Gly-14.

Experimental results. Buckminsterfullerenes do not affect mechanosensitivity of MscL. Open probability $\left(\mathrm{P}_{\mathrm{o}}\right)$ values were derived using the Event Detection tool on Clampfit from recordings showing only single channel currents, which were fitted to a Boltzmann distribution function using Prism 6 graphic program (GraphPad Software, Inc., CA). These experimental values were plotted against their corresponding pressures at which the recording was taken, which were then converted to membrane tension using the relationship between half activation and its respective membrane tension $\mathrm{P}_{1 / 2}=12 \mathrm{mN} / \mathrm{m}^{20,21}$. There were no significant differences between the channel open probabilities of the treated and control MscL (as shown in Supplementary Figure 1), suggesting that the channel mechanosensitivity was not affected by exposure to the $\mathrm{C}_{60}$ complex.

The maximum single channel current for each voltage level was measured for treated and control samples and plotted in Figure 6. Since the slope of the current-voltage plot is reduced at both positive and negative voltages for the treated MscL channels it is an indication of a reduction in the conductance of both inward and outward channel currents. The conductance value is $3.35 \mathrm{nS}$ for the fully open channel current recorded for the untreated MscL and 2.99 nS for the treated MscL, respectively, which is consistent with the previously reported conductance values for WT $\mathrm{MscL}^{22,23}$. However, Figure 6 also shows a significant reduction in the average MscL conductance, which was caused by the addition of the fullerenecomplex. It should be noted that fully open channels were much rarer and had short open dwell times in the presence of the fullerene
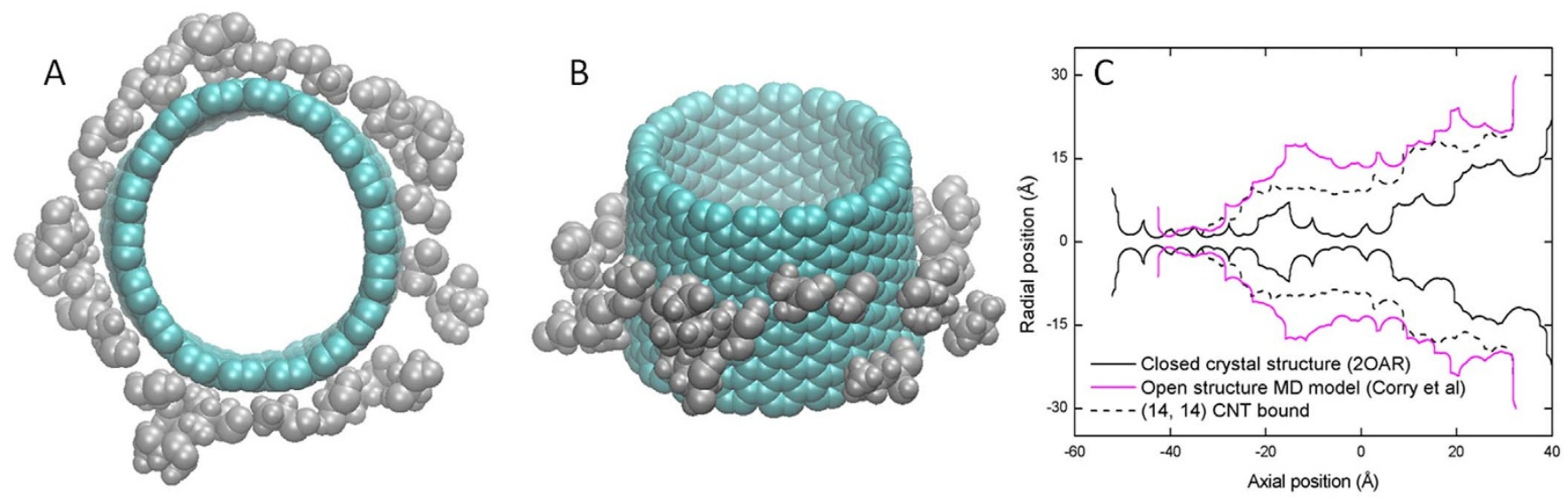

Figure $5 \mid(\mathrm{A})$ top view and (B) side view of the $(18,18)$ carbon nanotube $(\mathrm{CNT})$ within the MscL pore. Hydrophobic residues wrap around the nanotube surface, highlighted in grey. (C) MscL pore profile when the $(14,14)$ carbon nanotube is bound to the pore compared to the closed crystal structure $^{11,20,37}$, and open all-atom model ${ }^{17}$. 


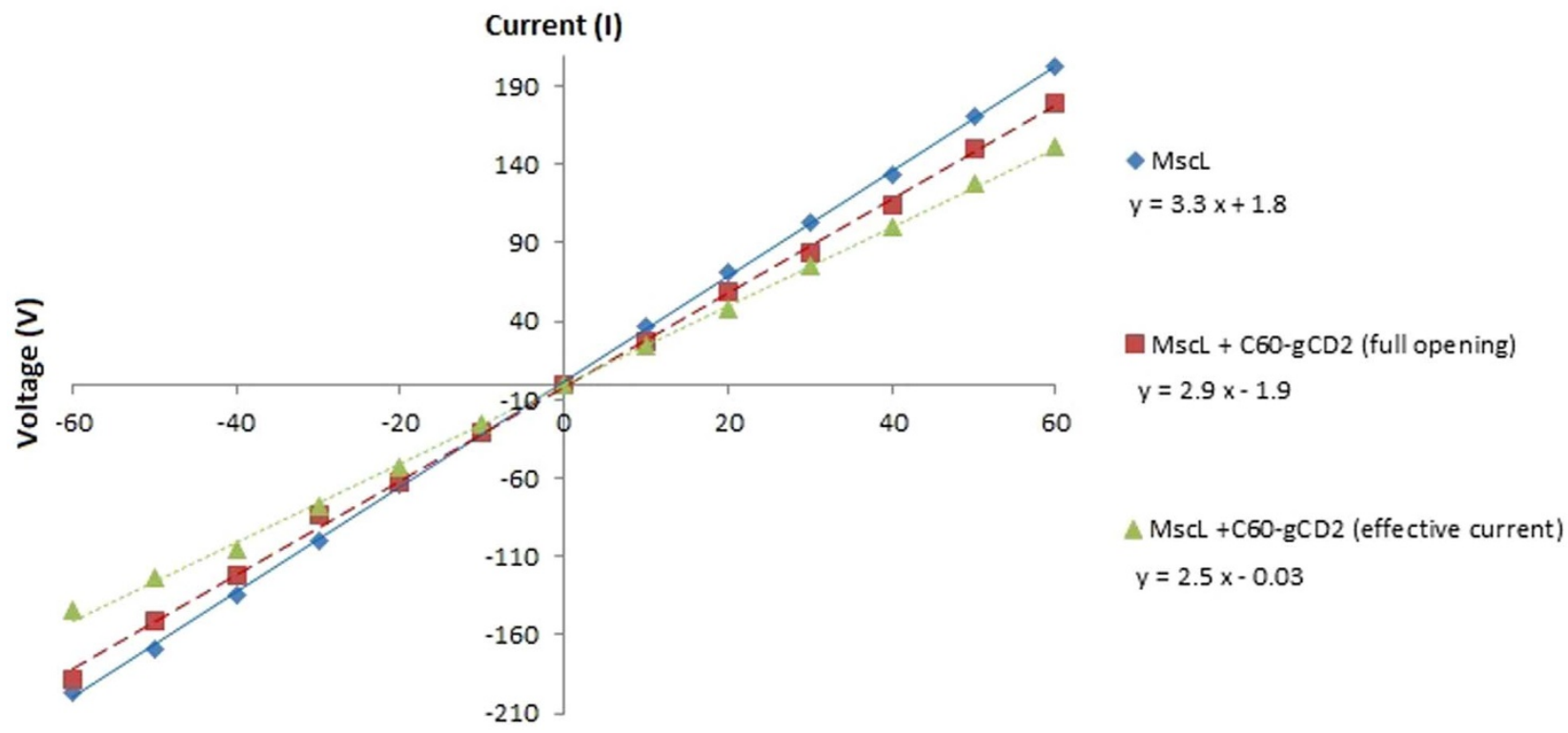

Figure $6 \mid$ Mean MscL current (pA) plotted against voltage $(\mathrm{mV})$ for the treated and control channels.

complex. Consequently, the I/V plot for the fully open channel in the presence of the complex represents a very small fraction of events recorded (traces shown in Figure 7). These open channel amplitudes were deduced from recordings where more than one channel was present.

The typical traces reported here show a marked difference in behaviour between the treated and untreated channels when stimulated with the same membrane tension. The treated channel exhibits preferential openings to sub-conducting levels rather than to the fully open state, which is reached at lower frequencies than in the control sample $(\sim 100 \mathrm{pA}$ at $+30 \mathrm{mV})$. Transitions between partially open and closed states are more frequent thus suggesting that the observed reduction in the channel conductance resulted from the fullerene complex being present. This reduction in channel conductance could be a result of partial blockage of the pore as observed in simulation. We have not measured the effect of fullerene on channel conductance in simulation; this will be the subject of our future work.

Buckminsterfullerene reduces current amplitude and dwell times. The amplitude histogram (Figure 8) shows a dramatic reduction in the number of full opening events (violet fit) in the channels treated with the fullerene complex when activated to $\sim 15 \%$ open probability. This is compensated by the increase in events where the channel opens to its sub-conducting levels; this marked change suggests an
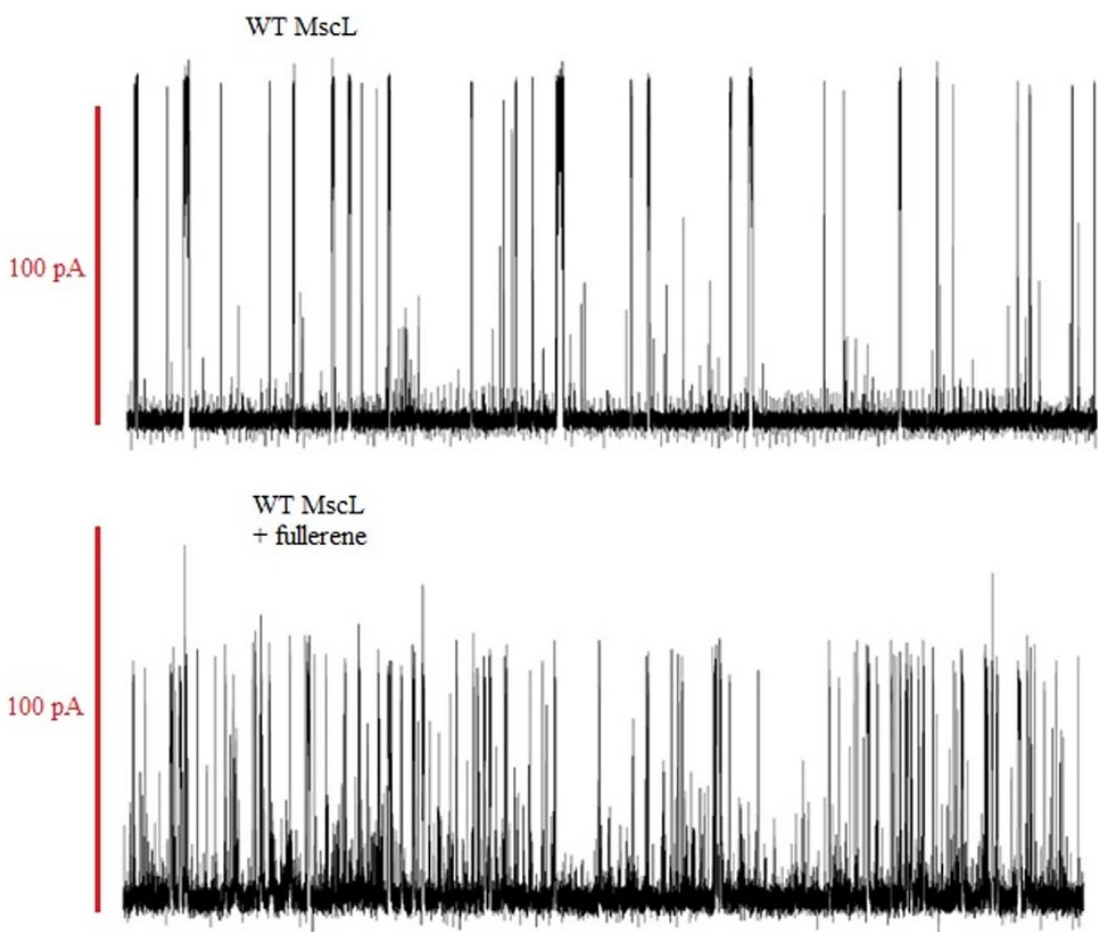

Figure $7 \mid$ Typical traces for the untreated (top) and treated (bottom) single channel MscL at $+30 \mathrm{mV}$. Both traces show channels having open probability of approximately $15 \%$. Red bar $=100 \mathrm{pA}$. Traces represent 3s-long recordings. 

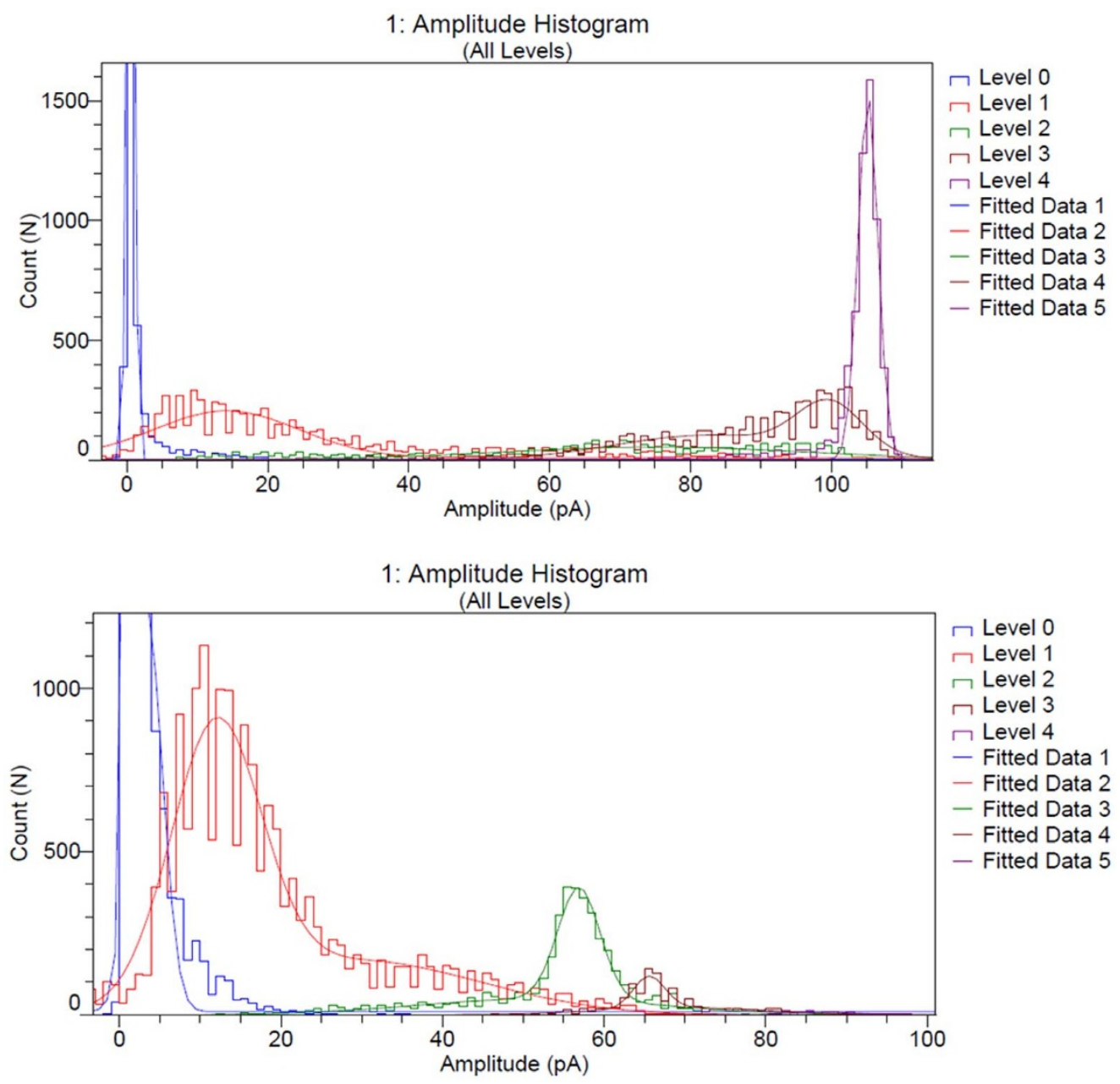

Figure 8 Amplitude histograms of the untreated (top) and treated (bottom) MscL (blue line = closed dwell time; red line = open dwell time green line $=$ opening to $\sim 60 \mathrm{pA}$ sub-conducting level, violet line $=$ full opening event). Open probability of the channels $\sim 15 \%$. Bin size $=0.5 \mathrm{pA}$.

interaction, or multiple interactions, between the fullerene complex and the pore of MscL. The complex appears to only partially block the current in a dynamic fashion as multiple sub-conducting level thresholds are crossed.

As also evident from the current traces in Figure 7, the MscL channels from the treated sample tended to spend less time in the closed state; this suggests that blockage of the pore prevents the channel from closing correctly (Figure 9). As the full opening events are rare, the violet line is not visible in the bottom histogram but is instead compensated by the green line which represents opening to the $\sim 60 \mathrm{pA}$ sub-conducting level. The graph also shows some very fast events at the lower sub-conducting levels of $\sim 20 \mathrm{pA}$, which in Figure 9 represent the majority of opening events. These events could either represent multiple fullerene complexes crowding the pore and therefore, reducing the ionic current by $>80 \%$ or possibly failed closing events that were prevented by a fullerene-complex obstructing the pore area.

\section{Discussion}

Fullerene interaction with MscL pore to partially block the ionic current. The experimental results demonstrate that the $C_{60}-\gamma$ cyclodextrin complex interacts with the pore and partially blocks its activity in a reversible fashion. Similarly, the simulation results demonstrate that both $\mathrm{C}_{60}$ and $\mathrm{C}_{60}(\mathrm{OH})_{24}$ interact with the pore and could partially block the flow of ions. Moreover, the fullerenes seem to disrupt the normal closure of the MscL pore which could be relevant when investigating any antimicrobial property of $\mathrm{C}_{60}$. Our simulations also demonstrate that more than one fullerene can interact with the pore, suggesting that multiple fullerenes could indeed block the pore altogether. Future MD studies with multiple fullerenes could simulate the event pore saturation. Calculating the free volume not blocked by the $\mathrm{C}_{60}$ complexes could help deduce the maximum current that could be blocked.

The $\mathrm{C}_{60}$ complex may also be interacting with the lipid bilayer surrounding MscL, since some phase exchange between complexed fullerenes and lipid bilayer has been reported elsewhere ${ }^{24,25}$. Cyclodextrin is known to remove detergent and cholesterol from bilayers. Therefore, the release of fullerenes in exchange for detergent would be possible (note that cholesterol was not present in our liposomes), but the unaffected mechanosensitive and kinetic properties of the channel (Supplementary Figure 1 and Figure 6) demonstrate this is unlikely. Analysing the stability of the complex when exposed to bilayers under physiological conditions would give us precious insight on the toxicity of bound fullerene with respect to free $\mathrm{C}_{60}$. It has previously been shown computationally that non-functionalized $\mathrm{C}_{60}$ fullerenes enter the hydrophobic interior of the lipid bilayer, but once functionalized the fullerenes will remain near the lipid head groups $^{26-28}$.

Both inward and outward currents were blocked by applying fullerenes on the cytosolic face of MscL. It is possible that the electrondense $\mathrm{C}_{60}$ would be repelled by the cathode within the pipette and therefore increased the blocking at negative voltages, but we found this was not the case. This could mean that once complexed with cyclodextrin the electronic properties of the nanomaterial are altered. 

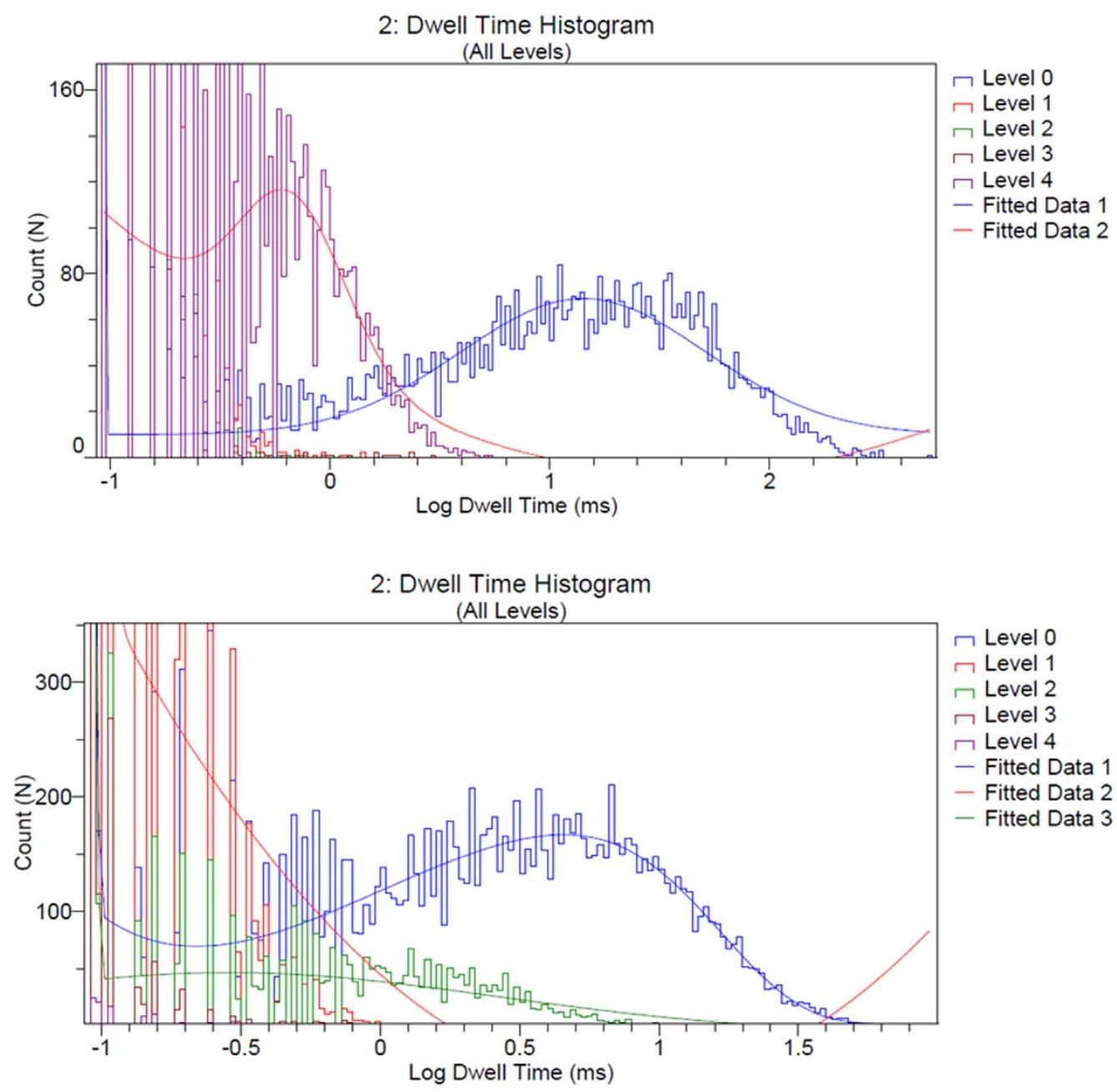

Figure $9 \mid$ Dwell time histograms of the untreated (top) and treated (bottom) MscL (blue line = closed dwell time; red line = open dwell time). Green line in the bottom histogram represents the open dwell time at the $\sim 60 \mathrm{pA}$ sub-conducting level. Open probability of the channels $\sim 15 \%$. Bin size $=0.5$.

The experimental results agree with the modelling on various aspects: the channel is not blocked to its full extent since the fullerenes are smaller than the pore; the channel is blocked in a dynamic way, multiple interacting residues can mean multiple fullerenes blocking at the same time and/or a single fullerene can diffuse to different sites and block to a different extent. In experiments this dynamicity in binding results in the channel being fully active in rare occasions. High pressure was found to trigger a quasi-full open state where the maximum current was limited to the last sub-conducting state before full opening (data not reported here) highlighting how heterogeneous binding to the pore could be. Since we were unable to control the stoichiometry of binding to the pore and to wash away any bound fullerenes we can only speculate.

Nanotube interacts with MscL pore to prevent pore closure. The simulation results demonstrate that both boron nitride and carbon nanotubes interact with the MscL pore, and prevent normal pore closure.

Relevance for the multidrug resistance. Multi-drug resistance in pathogenic strains of bacteria has, in the last decade, presented an increasing problem in the treatment of bacterial infections and diseases $^{8}$. For example, the re-emergence of tuberculosis is a serious threat that is spreading rapidly throughout the world ${ }^{8}$. It has become increasingly important to develop new antibiotics to combat the rapidly emerging strains of multiple drug-resistant bacterial strains. There is evidence to suggest that if MscL is gated inappropriately, such that it opens at low membrane tensions (or is forced to remain open), its presence is devastating for the cell ${ }^{\text {. For }}$ example, site-directed mutations of MscL suggested that disruption of MscL gating in gain-of-function (GOF) mutants severely inhibits cell growth ${ }^{12}$. It is possible that the highly conserved nature of MscL among prokaryotes (2296 members of the MscL subfamily identified to date in prokaryotic species, including many bacterial pathogen ${ }^{8,10}$ ) could be exploited for developing broad spectrum antimicrobials ${ }^{1}$. We have demonstrated the exciting potential of fullerenes and nanotubes as potential antimicrobial agents using MD simulations by showing that both fullerenes and nanotubes bind to the interior pore of MscL. From our simulations we predict that fullerenes will attenuate the ionic current through MscL, whereas nanotubes will prevent the closure of MscL once osmotic equilibrium is restored. We confirm experimentally that fullerenes interact with the MscL pore, and partially block the pore. It is likely that both fullerenes and nanotubes will cause MscL to malfunction so that it can no longer act as an effective emergency release valve. The addition of fullerenes would increase the time it takes for bacteria to reach osmotic equilibrium, and the addition of nanotubes would cause large current leakage to occur. Both of these events may be sufficient to cause bacterial cells to lyse. It is possible that the fullerenes and nanotubes examined here may be toxic to human cells by binding to proteins with hydrophobic surfaces or pockets. Unfortunately, this would result in an adverse effect to infected 
patients. Therefore, the toxicity of these compounds deserves further investigation.

Since the absence of either MscS or MscL can be compensated with the other $r^{7,8}$, it is important to consider whether the fullerene and nanotube would interact with both $\mathrm{MscL}$ and MscS. At their narrowest point, the pores are constricted by the side chains of symmetry-related residues: Leu-17 and Val-21 in MscL, and Leu-105 and Leu-109 in $\mathrm{MscS}^{11}$. Both channels also have a wide extracellular vestibule, so that once open, access to these hydrophobic residues could be made by an incoming fullerene or nanotube. It is likely that a fullerene or nanotube would also be attracted to the constriction of $\mathrm{MscS}$ as a result of these hydrophobic residues. Since the diameter of $\mathrm{MscS}$ is smaller than MscL the pore may be more quickly blocked by multiple $\mathrm{C}_{60}$ fullerenes. However, the use of nanotubes with diameters below the open diameter of $\operatorname{MscS}\left(14-16 \AA^{7}\right)$ would be an important design consideration for the use of nanotubes as antimicrobial agents.

In future work we hope to confirm the nanotube results experimentally. Experiments using the fullerenes examined here are possible since these fullerenes are readily available for purchase online (for example www.buckyusa.com). However, nanotubes with lengths $<100 \mathrm{~nm}$ are not yet commercially available.

We also hope to test the effect of fullerenes and nanotubes in vivo in live bacteria. Such a study would require the E. coli null mutant lacking all MS channels, and there are seven of them. The null mutant would have to carry MscL on a plasmid vector, so that it would only express MscL. This mutant could then be used to test in vivo the effects of fullerenes and nanotubes on solute and ion fluxes through activated MscL alone. Unfortunately, we cannot perform these experiments at present because of the unavailability of the E. coli MS channel null mutant expressing MscL alone. However, we plan to get this mutant in our future studies.

\section{Methods}

Simulation details. Fullerene and nanotube structures. We examined two fullerene structures in $\mathrm{MD}$ simulations, a $\mathrm{C}_{60}$ fullerene and $\mathrm{C}_{60}(\mathrm{OH})_{24}$ fullerene, as shown in Figure $10 \mathrm{~A}$ and $10 \mathrm{~B}$, respectively. The $\mathrm{C}_{60}$ fullerene structure was taken from the Nanotube Modeler PDB database [Nanotube Modeler v. 1.7.3 (JCrystalSoft, 20052012)] and the $\mathrm{C}_{60}(\mathrm{OH})_{24}$ structure was taken from the work of Fileti et al. ${ }^{29}$. Partial charges on the $\mathrm{C}_{60}(\mathrm{OH})_{24}$ fullerene were taken from the CHARMM36 force field ${ }^{30,31}$. All atoms of the fullerene were aromatic carbons with no charge unless attached to a hydroxyl group. The $\mathrm{C}_{60}$ and $\mathrm{C}_{60}(\mathrm{OH})_{24}$ fullerenes have approximate diameter of 7 and $11 \AA$, respectively.

We examined three nanotube structures in MD simulations, a $(18,18)$ boron nitride nanotube $(\mathrm{BNT})$, and a $(18,18)$ and $(14,14)$ carbon nanotube $(\mathrm{CNT})$ all with a length of approximately $20 \AA$. The $(18,18)$ CNT is illustrated in Figure 10C. The nanotubes were constructed using the basic relations governing the fundamental parameters of a CNT ${ }^{32}$ and a boron-nitrogen or carbon-carbon bond distance of 1.446 $\AA^{33,34}$ and $1.42 \AA^{32}$, respectively. The Lennard-Jones constants and partial charges $( \pm 0.4 \mathrm{e})$ for boron and nitrogen atoms of the BNT were taken from Won and Aluru $^{33,34}$. The carbon atoms on the CNTs were considered neutral and aromatic carbon parameters from the CHARMM36 force field were used ${ }^{30,31}$. The $(18,18)$ BNT, $(14,14) \mathrm{CNT}$, and $(18,18)$ CNT have approximate diameters of $24.8,19$, and $24 \AA$, respectively.

Molecular dynamics. All MD simulations were performed using NAMD 2.9 and visualized using VMD 1.9.1 $1^{35,36}$. All simulations used the CHARMM36 force field ${ }^{30,31}$ and TIP3P water with a time step of $2 \mathrm{fs}$, at constant pressure (1 atm) and temper-
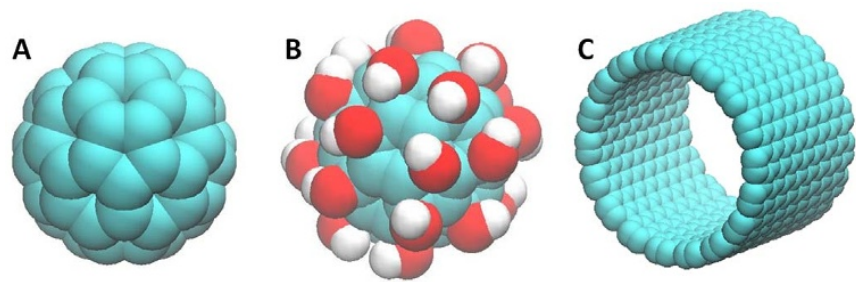

Figure $10 \mid$ Nanostructures. (A) $\mathrm{C}_{60}$ fullerene, (B) $\mathrm{C}_{60}(\mathrm{OH})_{24}$ fullerene, and $(\mathrm{C})(18,18)$ carbon nanotube. Note that the same scale has not been used for each part. ature $(310 \mathrm{~K})$. Each system was replicated periodically in all three dimensions and particle-mesh Ewald electrostatics was used. We performed equilibrium MD simulations of the two fullerene structures in both a closed and open MscL pore, and the three nanotubes on an open MscL pore only. The closed crystal structure of MscL from Mycobacterium tuberculosis was obtained from the protein database (PDB ID: 2OAR $)^{11,37}$, and the open structure was obtained from the work of Corry et al. ${ }^{17}$. The model of Corry et al. ${ }^{17}$ was generated using constrained MD simulations enforcing experimentally derived distances from fluorescence resonance energy transfer (FRET) and electron paramagnetic resonance spectroscopy studies on $\mathrm{MscL}^{38}$ cloned from Escherichia coli ${ }^{23}$. The MscL channel is pentameric, and in this paper the subunits are referred to as $\mathrm{CHA}, \mathrm{CHB}, \mathrm{CHC}, \mathrm{CHD}$ and $\mathrm{CHE}$. The MscL pore was embedded in a POPE lipid bilayer, solvated in a 120 by 120 by $120 \AA^{3}$ box of water. The entire simulation system is shown in Supplementary Figure 3. Potassium and chloride ions were added both to neutralize the system and to simulate an ionic concentration of $200 \mathrm{mM}$. The protein and fullerene/nanotube were initially held fixed, allowing the water, ions and lipid bilayer to equilibrate. This allowed the bilayer to equilibrate before the restraints on $\mathrm{MscL}$ were removed. For the remaining simulations, the protein and lipid bilayer centres of mass were held by a harmonic constraint of $0.2 \mathrm{kcal} \mathrm{mol}^{-1} \AA^{-2}$.

In simulations using the closed pore both fullerenes were placed at a distance of $(0$, 0,10 ), approximately $12 \AA$ from the Val-21 residue of the MscL channel which forms the narrowest constriction. Simulations were equilibrated for $1 \mathrm{~ns}$.

We ran four simulations, each lasting $6 \mathrm{~ns}$, using the open pore and the $\mathrm{C}_{60}$ fullerene placed on the pore central axis, at various $z$ positions, namely $z=0,5,10$, and $20 \AA$ A. Similarly, two simulations were performed, each lasting $6 \mathrm{~ns}$, using the open pore and the $\mathrm{C}_{60}(\mathrm{OH})_{24}$ fullerene placed on the pore central axis, at $z=0$, and $30 \AA$ Two additional simulations were run using the open pore with either two $\mathrm{C}_{60}$ fullerenes or two $\mathrm{C}_{60}(\mathrm{OH})_{24}$ fullerenes present in the upper reservoir, for 3 and $4 \mathrm{~ns}$, respectively. The centre of mass of each of the three nanotubes is placed at a distance of $(0,0,9)$, and each simulation is run for $4 \mathrm{~ns}$. To get a sense of simulation time required to observe pore closure of the open pore model we also ran in the absence of fullerenes/nanotubes for $32 \mathrm{~ns}$.

We generated approximate pore outlines so that we were able to compare our unrestrained MD simulation of the open pore with both the closed crystal structure $^{11,37}$, and the open model generated from FRET ${ }^{17}$. Pore outlines represent the dielectric interface between protein and water and were determined by assigning the protein atoms Born radii ${ }^{39}$ and tracing the channel pore with a water molecule sphere of radius $1.4 \AA^{40}$ to generate the minimum boundary radius at each axial position.

We have not determined the binding energy for the fullerenes and nanotubes as this is beyond the scope of this work. We are hoping to determine a potential of mean force for both fullerenes and nanotubes in subsequent simulations.

Experimental details. Preparation of $C_{60} / \gamma$-Cyclodextrin complex. Initial experiments used non-functionalized fullerenes and polyhydroxy fullerenes with $18-22 \mathrm{OH}$ groups (Fullerene $\mathrm{C}_{60}$, BU-604, BuckyUSA, Houston, TX; Polyhydroxyl-C60 $\left(\mathrm{C}_{60}(\mathrm{OH})_{\mathrm{n}}, \sim \mathrm{n}=18-22, \mathrm{BD}-301\right.$, BuckyUSA, Houston, TX). The solubility of these samples was prohibitive as only ethanol could be chosen to maintain the integrity of MscL. As such, a fullerene/cyclodextrin complex was used in experiments as it had a better solubility. The fullerene/cyclodextrin complex was prepared as previously described (Komatsu et al., 1999). Dry amounts of reagents (Fullerene $\mathrm{C}_{60}$, BU-604, BuckyUSA, Houston, TX; $\gamma$-Cyclodextrin, C4892-1G Sigma)] were shaken in a stainless steel capsule containing a stainless steel ball at $2800 \mathrm{rpm}$ for 10 minutes. The resulting powder was scraped off the walls of the capsule and resuspended in $4 \mathrm{ml}$ of the pipette solution for patch clamp experiments $\left(200 \mathrm{mM} \mathrm{KCl}, 40 \mathrm{mM} \mathrm{MgCl}_{2}\right.$, 5 mM HEPES, pH 7.2 adjusted with $\mathrm{KOH}$ ). The resuspended solution (cloudy, brown colour) was then filtered using a hydrophobic $0.2 \mu \mathrm{m}$ syringe filter, which yielded a clear solution coloured magenta. The latter was then analysed with a spectrophotometer [ND-1000, Nanodrop, USA] by measuring its UV absorption spectra at $330 \mathrm{~nm}$ (Supplementary Figure 2) and compared with the reference ${ }^{41}$ spectra to confirm the presence of a fullerene/cyclodextrin complex. The concentration of the complex was calculated using Beer-Lambert Law and the extinction coefficient published previously ${ }^{41}$. The estimated concentration of the complex was $0.33 \mathrm{mM}$.

Liposome preparation. Liposomes made of DOPE/DOPC $[7: 3, \mathrm{w}: \mathrm{w}]$ were produced using the sucrose method ${ }^{42}$. Briefly, the lipids (Avanti) in chloroform were mixed and dried with a $\mathrm{N}_{2}$ gas stream until a thin film of lipid was formed on the inner wall of a glass tube. $1 \mathrm{ml}$ of $0.4 \mathrm{M}$ sucrose solution was then added to the tube and incubated at $55^{\circ} \mathrm{C}$ for 3 hours. Purified MscL protein was then added in a 1/4000 [w:w] ratio after a lipid cloud was formed in the liposome suspension and the sample cooled down to room temperature. Each tube was then left on a shaker overnight and stored at $4^{\circ} \mathrm{C}$ for up to 2 weeks for patch clamp experiments.

Patch clamp experiments. The patch clamp experiments aimed to compare changes in mechanosensitivity, conductance and gating kinetics between MscL treated with the fullerene/cyclodextrin complex and control MscL. The magenta solution containing the $\mathrm{C}_{60}$ complex was used as the pipette solution for the treated samples, while a clear pipette solution not containing the complex was used as a control. $\mathrm{C}_{60}$ complex was added to the pipette solution because of the right-side-out orientation of MscL in liposome patches ${ }^{43}$ meaning that the complex could enter the channel from its periplasmic extracellular side. Glass pipettes [Drummond Scientific, Broomall, PA] were pulled fresh before every experiment using a Narishige gravity puller [PP-83; 
Narishige, Tokyo, Japan]. Pressure was applied manually to the patch pipette using a syringe in a step-wise fashion as well as by applying pressure ramps using a highpressure clamp apparatus (HSPC-1 ALA Scientific Instruments, USA) and the channel current was recorded at $+30 \mathrm{mV}$ in order to plot open probability against the membrane tension. The channel current was also recorded in the range from $-60 \mathrm{mV}$ to $+60 \mathrm{mV}$ to obtain the current/voltage (I/V) plot. Currents were amplified with an AxoPatch 1D amplifier (Axon Instruments), and data were acquired at a sampling rate of $5 \mathrm{kHz}$ with $2-\mathrm{kHz}$ filtration.

Data analysis. Current recordings were analysed using the pClamp 10 analysis software (Molecular Devices, Sunnyvale, CA). The maximum currents were deduced from amplitude histograms created by the Clampfit software whenever sufficient numbers of full opening events were available for curve fitting; these were otherwise measured by eye if the number of full openings was particularly rare within a trace. The event detection tool of the suite allowed to count opening events at different amplitude thresholds, arbitrarily chosen based on the average sub-conducting level steps that have been previously reported for the wild-type MscL channels. These threshold gaps were kept unchanged, but had to be occasionally adjusted with respect to the baseline between the analyses of treated and untreated channels. The amplitude and dwell time histograms reported here were taken from typical traces with similar open probability values (12-15\%) in order to have a fair comparison between the treated and untreated samples. Open probability data was plotted on Graphpad Prism 6 , and curves were fitted to the experimental data using Boltzmann distribution functions.

1. Booth, I. R. \& Blount, P. The MscS and MscL families of mechanosensitive channels act as microbial emergency release valves. J. Bacteriol. 194, 4802-4809 (2012).

2. Kung, C., Martinac, B. \& Sukharev, S. Mechanosensitive channels in microbes. Annu. Rev. Microbiol. 64, 313-329 (2010).

3. Corry, B. \& Martinac, B. Bacterial mechanosensitive channels: experiment and theory. Biochim. Biophys. Acta 1778, 1859-1870 (2008).

4. Martinac, B. in Biological membrane ion channels: Dynamics, structure and applications (eds Chung, S. H., Andersen, O. S. \& Krishnamurthy, V.) (Springer, 2007).

5. Cruickshank, C. C., Minchin, R. F., Dain, A. C. L. \& Martinac, B. Estimation of the pore size of the large-conductance mechanosensitive ion channel of Escherichia coli. Biophys. J. 73, 1925-1931 (1997).

6. Sachs, F. Stretch-activated ion channels: what are they? Physiology 25, 50-56 (2010).

7. Naismith, J. H. \& Booth, I. R. Bacterial mechanosensitive channels-MscS: Evolution's solution to creating sensitivity in function. Annu. Rev. Biophys. 41 157-177 (2012).

8. Kloda, A. et al. Mechanosensitive channel of large conductance. Int. J. Biochem. Cell B. 40, 164-169 (2008)

9. Gottlieb, P. A., Suchyna, T. M., Ostrow, L. W. \& Sachs, F. Mechanosensitive ion channels as drug targets. Current Drug Targets - CNS \& Neurological Disorders 3 , 61-72 (2004).

10. Martinac, B. et al. Bacterial Mechanosensitive Channels: Models for Studying Mechanosensory Transduction. Antioxidants \& redox signaling, doi:10.1089/ ars.2013.5471 (2013)

11. Steinbacher, S., Bass, R., Strop, P. \& Rees, D. C. Structures of the prokaryotic mechanosensitive channels MscL and MscS. Curr. Top. Membr. 58, 1-24 (2007).

12. Blount, P., Schroeder, M. J. \& Kung, C. Mutations in a bacterial mechanosensitive channel change the cellular respons to osmotic stress. J. Biol. Chem. 272, 32150-32157 (1997).

13. Nguyen, T., Clare, B., Guo, W. \& Martinac, B. The effects of parabens on the mechanosensitive channels of E. coli. Eur. Biophys. J. 34, 389-395 (2005).

14. Kamaraju, K., Gottlieb, P. A., Sachs, F. \& Sukharev, S. Effects of GsMTx4 on bacterial mechanosensitive channels in inside-out patches from giant spheroplasts. Biophys. J. 99, 2870-2878 (2010).

15. Chen, R. \& Chung, S. H. Effect of gating modifier toxins on membrane thickness: implications for toxin effect on gramicidin and mechanosensitive channels. Toxins 5, 456-471 (2013).

16. Boulos, R. A. Antimicrobial dyes and mechanosensitive channels. Antonie van Leeuwenhoek 104, 155-167, doi:10.1007/s10482-013-9937-x (2013).

17. Corry, B. et al. An improved open-channel structure of MscL determined from FRET confocal microscopy and simulation. J. Gen. Physiol. 136, 483-494 (2010).

18. Corry, B., Rigby, P., Liu, Z. W. \& Martinac, B. Conformational changes involved in MscL channel gating measured using FRET spectroscopy. Biophys. J. 89, L49-L51 (2005).

19. Sotomayor, M. \& Schulten, K. Molecular dynamics study of gating in the mechanosensitive channel of small conductance MscS. Biophys. J. 87, 3050-3065 (2004).

20. Sukharev, S. I., Sigurdson, W. J., Kung, C. \& Sachs, F. Energetic and spatial parameters for gating of the bacterial large conductance mechanosensitive channel, MscL. The Journal of general physiology 113, 525-540 (1999).

21. Nomura, T. et al. Differential effects of lipids and lyso-lipids on the mechanosensitivity of the mechanosensitive channels MscL and MscS Proceedings of the National Academy of Sciences of the United States of America 109, 8770-8775, doi:10.1073/pnas.1200051109 (2012).
22. Hamill, O. P. \& Martinac, B. Molecular basis of mechanotransduction in living cells. Physiological reviews 81, 685-740 (2001).

23. Sukharev, S. I., Blount, P., Martinac, B., Blattner, F. R. \& Kung, C. A largeconductance mechanosensitive channel in E. coli encoded by mscL alone. Nature 368, 265-268, doi:10.1038/368265a0 (1994).

24. Ikeda, A. et al. Direct and short-time uptake of [70]fullerene into the cell membrane using an exvchange reaction from a [70]fullerene- $\gamma$-cyclodextrin complex and the resulting photodynamic activity. Chem. Commun. 1547-1549 (2009).

25. Ikeda, A., Kawai, Y., Kikuchi, J. \& Akiyama, M. Effect of phase transition temperature of liposomes on preparation of fullerene-encapsulation liposomes by the fullerene-exchange reaction. Chem. Commun. 46, 2847-2849 (2010).

26. Qiao, R., Roberts, A. P., Mount, A. S., Klaine, S. J. \& Ke, P. C. Translocation of $\mathrm{C}_{60}$ and its derivatives across a lipid bilayer. Nano Lett. 7, 614-619 (2007).

27. Wong-Ekkabut, J. et al. Computer simulation study of fullerene translocation through lipid membranes. Nature Nanotech. 3, 363-368 (2008).

28. Li, L., Davande, H., Bedrov, D. \& Smith, G. D. A molecular dynamics simulation study of $\mathrm{C}_{60}$ fullerenes inside a dimyristoylphosphatidylcholine lipid bilayer. J. Phys. Chem. B 111, 4067-4072 (2007).

29. Fileti, E. E., Rivelino, R., Mota, F. d. B. \& Malaspina, T. Effects of hydroxyl group distribution on the reactivity, stability and optical properties of fullerenols. Nanotechnology 19, 365703 (2008).

30. MacKerell, A. D., Jr, Feig, M. \& Brooks, C. L., III. Extending the treatment of backbone energetics in protein force fields: Limitations of gas-phase quantum mechanics in reproducing protein conformational distributions in molecular dynamics simulations. J. Comput. Chem. 25, 1400-1415 (2004).

31. MacKerell, A. D., Jr., Bashford, D., Bellott, M., Dunbrack, R. L. et al. All-atom empirical potential for molecular modeling and dynamic studies of proteins. J. Phys. Chem. B 102, 3586-3616 (1998).

32. Dresselhaus, M. S., Dresselhaus, G. \& Saito, R. Physics of carbon nanotubes. Carbon 33, 883-891 (1995).

33. Won, C. Y. \& Aluru, N. R. Structure and dynamics of water confined in a boron nitride nanotubes. J. Phys. Chem. C 112, 1812-1818 (2008).

34. Won, C. Y. \& Aluru, N. R. Water permeation through a subnanometer boron nitride nanotube. J. Am. Chem. Soc. 129, 2748-2749 (2007).

35. Humphrey, W., Dalke, A. \& Schulten, K. VMD: Visual molecular dynamics. J. Mol. Graphics 14, 33-38 (1996).

36. Phillips, J. C. et al. Scalable molecular dynamics with NAMD. J. Comput. Chem. 26, 1781-1802 (2005).

37. Chang, G., Spencer, R. H., Lee, A. T., Barclay, M. T. \& Rees, D. C. Structure of the MscL homolog from Mycobacterium tuberculosis: A gated mechanosensitive ion channel. Science 282, 2220-2226 (1998).

38. Perozo, E., Cortes, D. M., Sompornpisut, P., Kloda, A. \& Martinac, B. Open channel structure of MscL and the gating mechanism of mechanosensitive channels. Nature 418, 942-948, doi:10.1038/nature00992 (2002).

39. Nina, M., Beglov, D. \& Roux, B. Atomic radii for continuum electrostatic calculations based on molecular dynamics free energy simulations. J. Phys. Chem. B 101, 5239-5248 (1997).

40. Chung, S. H., Allen, T. W. \& Kuyucak, S. Modeling diverse range of potassium channels with Brownian dynamics. Biophys. J. 83, 263-277 (2002).

41. Komatsu, K., Fujiwara, K., Murata, Y. \& Braun, T. Aqueous solubilization of crystalline fullerenes by supramolecular complexation with $\gamma$-cyclodextrin and sulfocalix[8] arene under mechanochemical high-speed vibration milling. J. Chem. Soc., Perkin 1, 2963-2966 (1999).

42. Battle, A. R., Petrov, E., Pal, P. \& Martinac, B. Rapid and improved reconstitution of bacterial mechanosensitive ion channel proteins MscS and MscL into liposomes using a modified sucrose method. FEBS letters 583, 407-412, doi:10.1016/j.febslet.2008.12.033 (2009).

43. Ajouz, B., Berrier, C., Besnard, M., Martinac, B. \& Ghazi, A. Contributions of the different extramembranous domains of the mechanosensitive ion channel MscL to its response to membrane tension. The Journal of biological chemistry $\mathbf{2 7 5}$, 1015-1022 (2000).

\section{Acknowledgments}

We thank Ben Corry for his scientific advice and for providing us with an open pore model of MscL. We thank Rong Chen and Michael Thomas for their scientific advice. This work was supported by the NCI National Facility at the Australian National University. We gratefully acknowledge the support from the Australian Research Council through a Discovery Early Career Researcher Award, and the National Health and Medical Research Council.

\section{Author contributions}

T.A.H. and P.R. wrote the main manuscript text. T.A.H. prepared figures $1-5,10$ and P.R. prepared figures 6-9 and supplementary figures. T.A.H. conducted the simulations, T.A.H. and S.H.C. analysed the results. P.R., Y.N. and B.M. conducted the experiments and analysed the corresponding results. All authors reviewed and edited the manuscript. 


\section{Additional information}

Supplementary information accompanies this paper at http://www.nature.com/ scientificreports

Competing financial interests: The authors declare no competing financial interests.

How to cite this article: Hilder, T.A., Ridone, P., Nakayama, Y., Martinac, B. \& Chung, S.-H. Binding of fullerenes and nanotubes to MscL. Sci. Rep. 4, 5609; DOI:10.1038/srep05609 (2014).
This work is licensed under a Creative Commons Attribution-NonCommercialShareAlike 4.0 International License. The images or other third party material in this article are included in the article's Creative Commons license, unless indicated otherwise in the credit line; if the material is not included under the Creative Commons license, users will need to obtain permission from the license holder in order to reproduce the material. To view a copy of this license, visit http:// creativecommons.org/licenses/by-nc-sa/4.0/ 